\title{
Software para reabilitaçáo auditiva de crianças com distúrbios do processamento auditivo central
}

\section{Carla Gentile Matas}

Fonoaudióloga, Profa. Assistente, Doutora do Curso de Fonoaudiologia do Departamento de Fisioterapia, Fonoaudiologia e Terapia Ocupacional da Faculdade de Medicina da Universidade de São Paulo, São Paulo-SP, Brasil.

A American Speech Language Hearing Association - $A S H A^{1}$ define o processamento auditivo central como a eficiência e eficácia com a qual o sistema nervoso central utiliza a informação auditiva, sendo que o processamento temporal auditivo refere-se à percepção das características temporais do som, ou a percepção na mudança da duração dessas características, dentro de um intervalo de tempo restrito. Sendo assim, a integridade do sistema nervoso auditivo central é condição primordial para que estas mudanças possam ser percebidas e, consequentemente, para que a informação acústica seja processada de maneira adequada.

O Transtorno do Processamento Auditivo é um grupo complexo e heterogêneo de alteraçôes geralmente associado a uma série de dificuldades auditivas e de aprendizado, porém na presença de sensitividade auditiva normal. Estas alteraçôes podem estar presentes nas habilidades de localização e lateralização sonora, discriminação e reconhecimento auditivo; aspectos temporais; resolução, mascaramento, integração e ordenação temporal ${ }^{2}$.

$\mathrm{Na}$ literatura especializada existem diversas evidências de que o treinamento auditivo, técnica amplamente utilizada na intervenção de indivíduos com transtorno de processamento auditivo, melhora as funções auditivas que se encontram prejudicadas, sendo tal aspecto fundamentado na plasticidade neural e evidenciado por meio dos Potenciais Evocados Auditivos $^{3,4}$.

Do ponto de vista clínico, os programas de treinamento auditivo desenvolvidos podem ser aplicados em cabine acústica mediante a utilização de estímulos acústicos modificados similares aos testes que avaliam o processamento auditivo, ou podem ser aplicados por meio de computador mediante o desenvolvimento de softwares específicos.

Murphy e Schochat ${ }^{5}$ desenvolveram um programa de treinamento auditivo computadorizado, aplicando-o e analisando a sua eficácia em crianças com dislexia. Concluíram que o software elaborado mos- trou-se eficaz para o treinamento auditivo temporal, o que pôde ser comprovado por meio da melhora pós treinamento em relação a esta habilidade.

Neste número, a Revista de Neurociências apresenta um estudo que tem como objetivo principal a verificação da eficácia do Software Auxiliar de Reabilitação de Distúrbios Auditivos (SARDA) no tratamento de crianças com distúrbios de processamento auditivo central, tendo sido demonstrado que o software em questáo mostrou-se eficiente como procedimento terapêutico ${ }^{6}$. Os autores ressaltam que, pensando-se em reabilitação auditiva, torna-se de suma importância a utilização de estratégias terapêuticas que sejam prazerosas e que propiciem a interação do sujeito em atividades lúdicas e motivadoras, sendo esta uma das vantagens do treinamento auditivo computadorizado, além do controle por parte do terapeuta dos estímulos e da hierarquia das atividades.

Sendo assim, este estudo alerta para a necessidade de mais pesquisas na área de treinamento auditivo, visando a melhora na qualidade de vida de crianças e/ ou adultos que apresentem transtorno do processamento auditivo.

\section{REFERÊNCIAS}

1.(Central) Auditory processing disorders. Technical report. (Endereço na Internet) USA: American Speech-Language-Hearing Association (ASHA) (atualizado em: 03/2005; citado em: 04/2005). Disponível em: http:// www.asha.org/members/deskref-journals/deskrefault.

2.Chermak GD, Musiek FE. Auditory training: principles and approaches for remediation and managing auditory processing disorders. Semin Hear 2002;23:297-308.

3.Tremblay K, Kraus N, Mc Gee T, Ponton C, Otis B. Central auditory plasticity: changes in N1-P2 complex after speech-sound training. Ear Hear 2001;22:79-90.

4.Jirsa RE. Clinical efficacy of electrophysiologic measures in APD management programs. Semin Hear 2002;23:349-55.

5.Murphy CFB, Schochat E. Effect of non-linguistic auditory training on phonological and reading skills. Folia Phoniatr Logop 2010;63:147-53.

6.Comerlatto Junior AA, Silva MP, Balen SA. Software para reabilitação auditiva de crianças com distúrbios no processamento auditivo central. Rev Neurocienc 2010;18:454-62. 